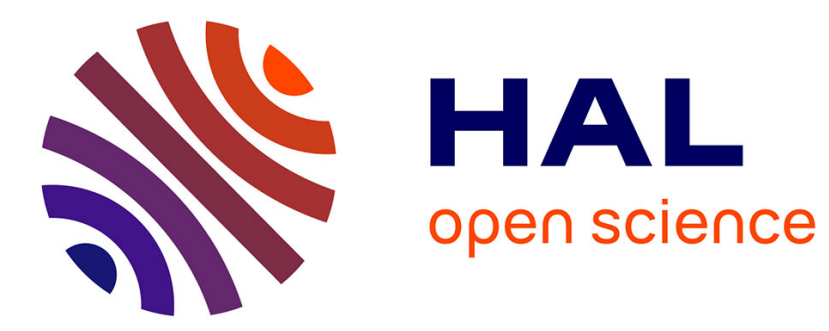

\title{
Model of two-temperature convective transfer in porous media
}

\author{
Isabelle Gruais, Dan Poliševski
}

\section{To cite this version:}

Isabelle Gruais, Dan Poliševski. Model of two-temperature convective transfer in porous media. Zeitschrift für Angewandte Mathematik und Physik, 2017, 68 (6), pp.article 143. 10.1007/s00033017-0889-2 . hal-01522808

\section{HAL Id: hal-01522808 \\ https://hal.science/hal-01522808}

Submitted on 15 May 2017

HAL is a multi-disciplinary open access archive for the deposit and dissemination of scientific research documents, whether they are published or not. The documents may come from teaching and research institutions in France or abroad, or from public or private research centers.
L'archive ouverte pluridisciplinaire HAL, est destinée au dépôt et à la diffusion de documents scientifiques de niveau recherche, publiés ou non, émanant des établissements d'enseignement et de recherche français ou étrangers, des laboratoires publics ou privés. 


\title{
Model of two-temperature convective transfer in porous media
}

\author{
Isabelle Gruais and Dan Poliševski
}

\begin{abstract}
In this paper, we study the asymptotic behaviour of the solution of a convective heat transfer boundary problem in an $\varepsilon$-periodic domain which consists of two interwoven phases, solid and fluid, separated by an interface. The fluid flow and its dependence with respect to the temperature are governed by the Boussinesq approximation of the Stokes equations. The tensors of thermal diffusion of both phases are $\varepsilon$-periodic, as well as the heat transfer coefficient which is used to describe the first-order jump condition on the interface. We find by homogenization that the two-scale limits of the solutions verify the most commonly system used to describe local thermal non-equilibrium phenomena in porous media (see [11] and [17]). Since now, this system was justified only by volume averaging arguments.
\end{abstract}

Mathematics Subject Classification (2010). 35B27; 76M50; 76Rxx; 74F10; 74Q05.

Keywords. Homogenization, $\varepsilon$-caps, Stokes-Boussinesq system, heat transfer coefficient, first-order jump interface, two-scale convergence.

\section{Introduction}

The macroscopic description of heat and mass transfer in porous media is of great interest in many geophysical and industrial problems. In the case of biphasic porous media, the one-temperature models imply a local thermal equilibrium between the solid and fluid phases. But this condition is not valid when the thermal properties differ widely or when the convective transport is important (see [5]). Thus, there have been considered the so-called non-equilibrium models, usually involving two temperatures. The first such models have been introduced heuristically (see [19]). Since now, these models were justified only by volume averaging arguments (see [16], [11] and [20]).

In this paper we find a two-temperature model for a convective heat and mass transfer model using the homogenization method. We still use the interfacial thermal barrier introduced by [16], reminding of a similar problem, that of the flow of slightly compressible fluids in porous media (see [2]), which was justified by homogenization in [6].

We study here the asymptotic behaviour of the solution of a convective heat transfer boundary problem in an $\varepsilon$-periodic domain which consists of two interwoven phases, solid and fluid, separated by an interface. The fluid part is connected and the only one reaching the boundary of the domain. The solid part is not necessarily connected.

We improve here the biphasic structure introduced in [15], by attaching at its ends the so-called cells of $\varepsilon$-caps, which allow the $\varepsilon$-periodic interface to be as smooth as it is needed. Moreover, all the properties of [15] hold for the present structure too.

We consider an incompressible viscous fluid flow which is governed by the Stokes equation, assuming that the temperature differences are small enough to allow the Boussinesq approximation of the Stokes equations. 
We consider that the tensors of thermal diffusion of the two phases are $\varepsilon$-periodic and not necessarily equal. On the interface, the heat flux is continuous and proportional with the temperature jump. This first-order jump condition presents an heat transfer coefficient which is also assumed $\varepsilon$ periodic. In order to balance the measure of the surface of the interface, we assume that the heat transfer coefficient is of $\varepsilon$-order. Finally, a temperature distribution is imposed on the boundary of the domain.

We prove the existence and uniqueness properties of the corresponding velocity, pressure and temperature distribution. An $L^{\infty}$-estimate of the temperature, uniform with respect to $\varepsilon$, is obtained.

The homogenization process is performed in the case when the Rayleigh number is of unity order. Using the techniques of the two-scale convergence theory (see [10], [1] and [3]), we find the twoscale system verified by the two-scale limits of the $\varepsilon$-solutions and the solutions of the local problems which allow us to define the effective coefficients of the homogenized system, called sometimes as the macroscopic system, as it does not depend on the microscopic variable.

The homogenized system that is verified here by the limits of the $\varepsilon$-solutions turns out to be the most common system used to describe local thermal non-equilibrium phenomena in porous media (see [16], [11] and [17]).

\section{The $\varepsilon$-caps of the periodic porous structure}

Let $\Omega$ be an open connected bounded set in $\mathbb{R}^{N}, N \geq 1$, locally located on one side of the boundary $\partial \Omega$, a Lipschitz manifold composed of a finite number of connected components.

Let $E$ be the rhombic polyhedron obtained by affixing square pyramids of $1 / 2$ height on each face of the cube $Y=]-1 / 2,1 / 2\left[^{N}\right.$, that is

$$
E=\operatorname{int}\left(\operatorname{Conv}\left(\bar{Y} \cup\left\{ \pm e_{i}, i=1,2, \ldots, N\right\}\right)\right),
$$

where $e_{i}$ are the unit vectors of the canonical basis in $\mathbb{R}^{N}$.

For $D \subset \subset E$, a domain which is of Lipschitz class, we define

$$
D_{i}^{ \pm}=\left(Y \pm e_{i}\right) \cap D, \quad \forall i \in\{1,2, \ldots, N\} .
$$

We assume that $Y_{s}:=Y \cap D$ has the property

$$
\bar{Y}_{s} \cap \Sigma^{ \pm i} \subset \subset \Sigma^{ \pm i}, \quad \forall i \in\{1,2, \ldots, N\},
$$

where $\Sigma^{ \pm i}=\left\{y \in \partial Y: y_{i}= \pm 1 / 2\right\}$.

Denoting $Y_{f}:=Y \backslash \bar{Y}_{s}$, we assume that the reunion of all the $\bar{Y}_{f}$ parts is a connected domain in $\mathbb{R}^{N}$ with a locally Lipschitz boundary, denoted by $\mathbb{R}_{f}^{N}$. Obviously, the origin of the coordinate system can be set in such a way that there exists $r>0$ with the property $B(0, r) \subseteq \mathbb{R}_{f}^{N}$.

For any $\varepsilon \in] 0,1[$ we denote

$$
\begin{gathered}
\mathbb{Z}_{\varepsilon}=\left\{k \in \mathbb{Z}^{N}: \varepsilon k+\varepsilon Y \subseteq \Omega\right\}, \\
\mathbb{I}_{\varepsilon}=\left\{k \in \mathbb{Z}_{\varepsilon}: \varepsilon k \pm \varepsilon e_{i}+\varepsilon Y \subseteq \Omega, \forall i \in\{1,2, \ldots, N\}\right\}, \\
\Omega_{Y_{\varepsilon s}}=\operatorname{int}\left(\bigcup_{k \in I_{\varepsilon}}\left(\varepsilon k+\varepsilon \bar{Y}_{s}\right)\right) \\
\mathbb{J}_{\varepsilon, k}^{ \pm}=\left\{i \in\{1,2, \ldots, N\}, \quad\left(\varepsilon k+\varepsilon \bar{D}_{i}^{ \pm}\right) \cap \Omega_{Y_{\varepsilon, s}} \neq \emptyset\right\}, \quad \forall k \in \mathbb{Z}_{\varepsilon} \backslash \mathbb{I}_{\varepsilon} .
\end{gathered}
$$

For every $k \in \mathbb{Z}_{\varepsilon} \backslash \mathbb{I}_{\varepsilon}$ we define the so-called cell of $\varepsilon$-caps by

$$
D_{\varepsilon, k}=\left(\cup_{i \in \mathbb{J}_{\varepsilon, k}^{+}}\left(\varepsilon k+\varepsilon D_{i}^{+}\right)\right) \cup\left(\cup_{i \in \mathbb{J}_{\varepsilon, k}^{-}}\left(\varepsilon k+\varepsilon D_{i}^{-}\right)\right) \subset \varepsilon k+\varepsilon Y .
$$

Thus, the solid part of our structure is given by

and the fluid part by

$$
\Omega_{\varepsilon, s}=\operatorname{int}\left(\Omega_{Y_{\varepsilon s}} \cup\left(\cup_{k \in \mathbb{Z}_{\varepsilon} \backslash \mathbb{I}_{\varepsilon}} D_{\varepsilon k}\right)\right)
$$

$$
\Omega_{\varepsilon f}=\Omega \backslash \bar{\Omega}_{\varepsilon s} .
$$


The interface between the two components is denoted by

$$
\Gamma_{\varepsilon}=\partial \Omega_{\varepsilon f} \cap \partial \Omega_{\varepsilon s}=\partial \Omega_{\varepsilon s} .
$$

We see that all the boundaries are at least locally Lipschitz, $\Omega_{\varepsilon f}$ is connected and $\Omega_{\varepsilon s}$ can be, in particular, connected too. We remark also that the cells of $\varepsilon$-caps are of at most $\left(4^{N}-2\right)$ types and that they do not affect the classical results concerning such $\varepsilon$-periodic structures (see [4], [6] and [15]). The main advantage of using the structure with cells of $\varepsilon$-caps is that $\Gamma_{\varepsilon}$ has the same class as the boundaries of $D$ and $\mathbb{R}_{f}^{N}$, which can be assumed as smooth as it is needed.

From now on, let us denote $\Gamma=\partial Y_{f} \cap \partial Y_{s}$ and $\nu$ the normal on $\Gamma$ (exterior to $Y_{f}$ ). Then, for $x \in(\varepsilon k+\varepsilon \Gamma), k \in \mathbb{Z}_{\varepsilon}$, we find that $\nu^{\varepsilon}(x)=\nu(\{x / \varepsilon\})$ is the corresponding normal on $\Gamma_{\varepsilon}$ (exterior to $\left.\Omega_{\varepsilon f}\right)$, where $\{x / \varepsilon\}$ stands for the fractional parts of the components of $\varepsilon^{-1} x$.

Our domain has the following well-known properties (see [4] and [6]):

Lemma 2.1. There exists an extension operator $P_{\varepsilon k} \in \mathcal{L}\left(H^{1}\left(\Omega_{\varepsilon k}\right) ; H^{1}(\Omega)\right)$, for any $k \in\{s, f\}$, with the properties:

$$
\begin{gathered}
P_{\varepsilon k} T=T \text { in } \Omega_{\varepsilon k}, \\
\left|\nabla P_{\varepsilon k} T\right|_{L^{2}(\Omega)} \leq C|\nabla T|_{L^{2}\left(\Omega_{\varepsilon k}\right)}, \forall T \in H^{1}\left(\Omega_{\varepsilon k}\right),
\end{gathered}
$$

where $C>0$ is a constant independent of $\varepsilon$.

Lemma 2.2. For any $T \in H_{0}^{1}\left(\Omega \backslash \Gamma_{\varepsilon}\right)$ there exists $C>0$, independent of $\varepsilon$, such that

$$
\begin{gathered}
|T|_{L^{2}\left(\Omega_{\varepsilon f}\right)} \leq C|\nabla T|_{L^{2}\left(\Omega_{\varepsilon f}\right)}, \\
\varepsilon^{1 / 2}\left|\gamma_{\varepsilon k} T\right|_{L^{2}\left(\Gamma_{\varepsilon}\right)} \leq C\left(|T|_{L^{2}\left(\Omega_{\varepsilon_{k}}\right)}+\varepsilon|\nabla T|_{L^{2}\left(\Omega_{\varepsilon k}\right)}\right), \quad k \in\{s, f\} \\
|T|_{L^{2}\left(\Omega_{\varepsilon s}\right)} \leq C\left(\varepsilon^{1 / 2}\left|\gamma_{\varepsilon s} T\right|_{L^{2}\left(\Gamma_{\varepsilon}\right)}+\varepsilon|\nabla T|_{L^{2}\left(\Omega_{\varepsilon s}\right)}\right) .
\end{gathered}
$$

Remark 2.3. Taking in account the $L^{2}$-norm of the jump on $\Gamma_{\varepsilon}$ the results of the previous Lemma have an important consequence:

$$
|T|_{L^{2}\left(\Omega_{\varepsilon s}\right)} \leq C|T|_{H_{0}^{1}\left(\Omega \backslash \Gamma_{\varepsilon}\right)}, \forall T \in H_{0}^{1}\left(\Omega \backslash \Gamma_{\varepsilon}\right) .
$$

We recall here the main inequalities that hold in the vectorial case.

Lemma 2.4. There exists $C>0$ independent of $\varepsilon$ such that

$$
\begin{gathered}
|u|_{L^{2}\left(\Omega_{\varepsilon f}\right)} \leq C \varepsilon|\nabla u|_{L^{2}\left(\Omega_{\varepsilon f}\right)}, \quad \forall u \in H_{0}^{1}\left(\Omega_{\varepsilon f}\right)^{N} \\
|p|_{L^{2}\left(\Omega_{\varepsilon f}\right) / \mathbb{R}} \leq \frac{C}{\varepsilon}|\nabla p|_{H^{-1}\left(\Omega_{\varepsilon f}\right)}, \quad \forall p \in L^{2}\left(\Omega_{\varepsilon f}\right) . \\
|p|_{L^{2}\left(\Omega_{\varepsilon f}\right) / \mathbb{R}} \leq C|\nabla p|_{L^{2}\left(\Omega_{\varepsilon f}\right)}, \quad \forall p \in H^{1}\left(\Omega_{\varepsilon f}\right) .
\end{gathered}
$$

Denoting $\chi_{k}^{\varepsilon}$ and $\chi_{k}$ as the characteristic functions of respectively $\Omega_{\varepsilon k}$ and $Y_{k}$, we have a specific compactness result for the pressure type estimates in $H^{-1}\left(\Omega_{\varepsilon f}\right)$ (see [15]).

Theorem 2.5. If $\left(p_{\varepsilon}\right)_{\varepsilon} \in L^{2}\left(\Omega_{\varepsilon f}\right)$ has the property that for some constant $C>0$ there hold:

$$
\begin{aligned}
& \left.\left|\int_{\Omega_{\varepsilon f}} p^{\varepsilon}(x) d x\right| \leq C, \quad \forall \varepsilon \in\right] 0,1[, \\
& \left.\left|\nabla p^{\varepsilon}\right|_{H^{-1}\left(\Omega_{\varepsilon f}\right)} \leq C \varepsilon, \quad \forall \varepsilon \in\right] 0,1[,
\end{aligned}
$$

then there exists $p \in L^{2}(\Omega)$ such that, on some subsequence:

$$
\chi_{\varepsilon f} p^{\varepsilon} \stackrel{2}{\rightarrow} \chi_{f} p .
$$




\section{The heat and mass transfer across the interface}

In this section, we present the existence and uniqueness properties, as well as the a priori estimates of the solutions of the convection problem occuring in the $\varepsilon$-periodic structure introduced in the previous section. We consider that the viscous fluid flow takes place only in $\Omega_{\varepsilon f}$ and is governed by the Stokes system in the Boussinesq approximation. The heat transport throughout $\Omega$ presents an interfacial barrier on $\Gamma_{\varepsilon}$, where the temperature obeys to a first-order jump condition. The problem is completed by imposing a certain temperature distribution on the boundary of $\Omega$.

Let us introduce the Hilbert space

$$
H_{0}^{1}\left(\Omega \backslash \Gamma_{\varepsilon}\right)=\left\{T \in L^{2}(\Omega),\left.T\right|_{\Omega_{\varepsilon f}} \in H^{1}\left(\Omega_{\varepsilon f}\right),\left.T\right|_{\Omega_{\varepsilon s}} \in H^{1}\left(\Omega_{\varepsilon s}\right), T=0 \text { on } \partial \Omega\right\}
$$

endowed with the scalar product

$$
(T, S)_{H_{0}^{1}\left(\Omega \backslash \Gamma_{\varepsilon}\right)}=\int_{\Omega_{\varepsilon f}} \nabla T \nabla S+\int_{\Omega_{\varepsilon s}} \nabla T \nabla S+\varepsilon \int_{\Gamma_{\varepsilon}}[T][S]
$$

where $[T]=\gamma_{\varepsilon s} T-\gamma_{\varepsilon f} T$ and $\gamma_{\varepsilon f} T, \gamma_{\varepsilon s} T$ are the traces of $T$ on $\Gamma_{\varepsilon}$ defined in $H^{1}\left(\Omega_{\varepsilon f}\right)$ and $H^{1}\left(\Omega_{\varepsilon s}\right)$, respectively.

For any $\varepsilon \in] 0,1\left[\right.$ we introduce the jump transmission factor $\eta^{\varepsilon}(x)=\eta(x / \varepsilon)$ and the symmetric conductivities $a_{i j}^{\varepsilon f}(x)=a_{i j}^{f}(x / \varepsilon)$ and $a_{i j}^{\varepsilon s}(x)=a_{i j}^{s}(x / \varepsilon)$, where $a_{i j}^{f}, a_{i j}^{s} \in L_{\text {per }}^{\infty}(Y)$ and $\eta \in C_{\text {per }}^{1}(Y)$ have the property that there exists $\delta>0$ such that

$$
\begin{gathered}
\eta \geq \delta, \text { a.e. on } Y \\
a_{i j}^{k} \xi_{i} \xi_{j} \geq \delta \xi_{i} \xi_{i} \quad \forall \xi \in \mathbb{R}^{N}, \text { a.e. on } Y, \forall k \in\{s, f\} .
\end{gathered}
$$

Also, we consider $\alpha_{\varepsilon}>0, g \in L^{\infty}(\Omega)^{N}$ and $Q \in L^{2}(\Omega)$ to be respectively the Rayleigh number, the external body force and the heat source. The nondimensional system which governs the velocity $u^{\varepsilon}$, pressure $p^{\varepsilon}$, and temperature $\theta^{\varepsilon}$, is the following (see [14]):

$$
\begin{aligned}
\operatorname{div} u^{\varepsilon} & =0 \text { in } \Omega_{\varepsilon f}, \\
\varepsilon^{2} \Delta u^{\varepsilon}+\theta^{\varepsilon} g & =\nabla p^{\varepsilon} \text { in } \Omega_{\varepsilon f}, \\
\alpha_{\varepsilon} u^{\varepsilon} \nabla \theta^{\varepsilon}-\operatorname{div}\left(a^{\varepsilon f} \nabla \theta^{\varepsilon}\right) & =Q \text { in } \Omega_{\varepsilon f}, \\
-\operatorname{div}\left(a^{\varepsilon s} \nabla \theta^{\varepsilon}\right) & =Q \text { in } \Omega_{\varepsilon s}, \\
a_{i j}^{\varepsilon f} \frac{\partial \theta^{\varepsilon}}{\partial x_{j}} \nu_{i}^{\varepsilon}=a_{i j}^{\varepsilon s} \frac{\partial \theta^{\varepsilon}}{\partial x_{j}} \nu_{i}^{\varepsilon} & =\varepsilon \eta^{\varepsilon}\left(\gamma_{\varepsilon s} \theta^{\varepsilon}-\gamma_{\varepsilon f} \theta^{\varepsilon}\right) \text { on } \Gamma_{\varepsilon} \\
u^{\varepsilon} & =0 \text { on } \partial \Omega_{\varepsilon f}, \\
\theta^{\varepsilon} & =\tau \text { on } \partial \Omega,
\end{aligned}
$$

where $\tau \in H^{3 / 2}(\partial \Omega)$, $\inf _{x \in \partial \Omega} \tau=-1 / 2$ and $\sup _{x \in \partial \Omega} \tau=1 / 2$.

Because of the nonlinear inertial term, we pass to a homogeneous problem with the following precaution (see [12] or [13]):

Lemma 3.1. For any $h>0$, there exists $\tau_{h} \in H^{2}(\Omega)$ such that:

$$
\begin{gathered}
\tau_{h}=\tau \quad \text { on } \quad \partial \Omega \\
\left|S \nabla \tau_{h}\right|_{L^{2}(\Omega)} \leq h|\nabla S|_{L^{2}(\Omega)}, \quad \forall S \in H_{0}^{1}(\Omega) .
\end{gathered}
$$

Setting $T^{\varepsilon}=\theta^{\varepsilon}-\tau_{h}$, for some $h$ which will be chosen later, and defining

$$
V_{\varepsilon f}=\left\{v \in H_{0}^{1}\left(\Omega_{\varepsilon f}\right), \quad \operatorname{div} v=0 \quad \text { in } \quad \Omega_{\varepsilon f}\right\},
$$

the variational formulation of our problem is:

To find $\left(u^{\varepsilon}, T^{\varepsilon}\right) \in V_{\varepsilon f} \times H_{0}^{1}\left(\Omega \backslash \Gamma_{\varepsilon}\right)$, solution of the system

$$
\varepsilon^{2}\left(\nabla u^{\varepsilon}, \nabla v\right)_{\varepsilon f}-\left(\left(T^{\varepsilon}+\tau_{h}\right) g, v\right)_{\varepsilon f}=0, \quad \forall v \in V_{\varepsilon f}
$$




$$
\begin{gathered}
\left(a^{\varepsilon f} \nabla\left(T^{\varepsilon}+\tau_{h}\right), \nabla S\right)_{\varepsilon f}+\left(a^{\varepsilon s} \nabla\left(T^{\varepsilon}+\tau_{h}\right), \nabla S\right)_{\varepsilon s}+\varepsilon \int_{\Gamma_{\varepsilon}} \eta^{\varepsilon}\left[T^{\varepsilon}\right][S]+ \\
+\alpha_{\varepsilon}\left(u^{\varepsilon}, S \nabla\left(T^{\varepsilon}+\tau_{h}\right)\right)_{\varepsilon f}=(Q, S)_{L^{2}(\Omega)}, \quad \forall S \in H_{0}^{1}\left(\Omega \backslash \Gamma_{\varepsilon}\right)
\end{gathered}
$$

where $(\cdot, \cdot)_{\varepsilon k}$ denotes the scalar product in $L^{2}\left(\Omega_{\varepsilon k}\right)^{N}, k \in\{s, f\}$.

Defining $\mathcal{F}_{\varepsilon} \in\left(V_{\varepsilon f} \times H_{0}^{1}\left(\Omega \backslash \Gamma_{\varepsilon}\right)\right)^{\prime}$ by

$$
\mathcal{F}_{\varepsilon}(v, S):=\delta\left(\tau_{h} g, v\right)_{\varepsilon f}+(Q, S)_{L^{2}(\Omega)}-\left(a^{\varepsilon f} \nabla \tau_{h}, \nabla S\right)_{\varepsilon f}-\left(a^{\varepsilon s} \nabla \tau_{h}, \nabla S\right)_{\varepsilon s},
$$

we introduce a problem equivalent to $(3.15)-(3.16)$ :

To find $\left(u^{\varepsilon}, \theta^{\varepsilon}\right) \in V_{\varepsilon f} \times H_{0}^{1}\left(\Omega \backslash \Gamma_{\varepsilon}\right)$ such that

$$
\left\langle G\left(u^{\varepsilon}, \theta^{\varepsilon}\right),(v, \varphi)\right\rangle=\mathcal{F}_{\varepsilon}(\varphi) \text { for any }(v, \varphi) \in V_{\varepsilon f} \times H_{0}^{1}\left(\Omega \backslash \Gamma_{\varepsilon}\right),
$$

where the mapping $G: V_{\varepsilon f} \times H_{0}^{1}\left(\Omega \backslash \Gamma_{\varepsilon}\right) \rightarrow\left(V_{\varepsilon f} \times H_{0}^{1}\left(\Omega \backslash \Gamma_{\varepsilon}\right)\right)^{\prime}$ is defined by

$$
\begin{aligned}
& \langle G(u, \theta),(v, \varphi)\rangle=h \alpha_{\varepsilon} \varepsilon^{2}\left(\nabla u^{\varepsilon}, \nabla v\right)_{\varepsilon f}-h \alpha_{\varepsilon}\left(T^{\varepsilon} g, v\right)_{\varepsilon f}+\varepsilon \int_{\Gamma_{\varepsilon}} \eta^{\varepsilon}\left[T^{\varepsilon}\right][S] \\
& +\left(a^{\varepsilon f} \nabla T^{\varepsilon}, \nabla S\right)_{\varepsilon f}+\left(a^{\varepsilon s} \nabla T^{\varepsilon}, \nabla S\right)_{\varepsilon s}+\alpha_{\varepsilon}\left(u^{\varepsilon}, S \nabla\left(T^{\varepsilon}+\tau_{h}\right)\right)_{\varepsilon f}
\end{aligned}
$$

Remark 3.2. It is obvious from (3.17) that we already know that $h \alpha_{\varepsilon}>0$ is a suitable multiplier for the flow equation.

In order to prove the existence theorem for problem (3.17), we remind the following result of Gossez (see [7]):

Theorem 3.3. Let $X$ be a reflexive Banach space and $G: X \rightarrow X^{\prime}$ a continuous mapping between the corresponding weak topologies. If

$$
\frac{\langle G \varphi, \varphi\rangle}{|\varphi|_{X}} \rightarrow \infty \quad \text { as } \quad|\varphi|_{X} \rightarrow \infty
$$

then $G$ is a surjection.

Here it is the main result of this section.

Theorem 3.4. There exists $\left(u^{\varepsilon}, T^{\varepsilon}\right)$ solution of the problem (3.15)-(3.16) for a proper choice of $h>0$, that is when $h \alpha_{\varepsilon}$ is smaller than a certain constant, independent of $\varepsilon$. Moreover, $T^{\varepsilon} \in L^{\infty}(\Omega)$ and there exists $c>0$ (independent of $\varepsilon$ ) such that

$$
\left|T^{\varepsilon}+\tau_{h}\right|_{L^{\infty}\left(\Omega_{\varepsilon f}\right)} \leq \frac{1}{2}+c|Q|_{L^{2}(\Omega)} .
$$

Proof. Using Theorem 3.3 and acting as in the proof of Theorem 5.2.2 [13] (Ch 1, Sec. 5), we obtain the first part of the result in a classical way.

In order to prove (3.20) let us define for any $t>0$

$$
\begin{gathered}
\Omega_{\varepsilon}^{t}=\left\{x \in \Omega,\left|T^{\varepsilon}(x)+\tau_{h}(x)\right|>1 / 2+t\right\}, \\
\Omega_{\varepsilon k}^{t}=\Omega_{\varepsilon}^{t} \cap \Omega_{\varepsilon k}, \quad k \in\{s, f\}, \\
R_{t}^{\varepsilon}=\operatorname{sgn}\left(T^{\varepsilon}+\tau_{h}\right) \max \left\{\left|T^{\varepsilon}+\tau_{h}\right|-1 / 2-t, 0\right\},
\end{gathered}
$$

where the inequalities are in the $H^{1}$-sense (see [9]). We see that

$$
R_{t}^{\varepsilon} \in H_{0}^{1}\left(\Omega \backslash \Gamma_{\varepsilon}\right) \text { and } \nabla R_{t}^{\varepsilon}=\nabla\left(T^{\varepsilon}+\tau_{h}\right) \text { in } L^{2}\left(\Omega \backslash \Gamma_{\varepsilon}\right) .
$$

As for any $k \in\{s, f\}$ we have

$$
\begin{gathered}
\left|R_{t}^{\varepsilon}\right|_{L^{4}\left(\Omega_{\varepsilon k}^{t}\right)}^{2} \leq\left|P_{\varepsilon k} R_{t}^{\varepsilon}\right|_{L^{4}(\Omega)}^{2} \leq c\left|\nabla P_{\varepsilon k} R_{t}^{\varepsilon}\right|_{L^{2}(\Omega)}^{2} \leq c\left|\nabla R_{t}^{\varepsilon}\right|_{L^{2}\left(\Omega_{\varepsilon k}^{t}\right)}^{2}= \\
=c\left|\nabla\left(T^{\varepsilon}+\tau_{h}\right)\right|_{L^{2}\left(\Omega_{\varepsilon k}\right)}^{2} \leq c\left(a^{\varepsilon k} \nabla\left(T^{\varepsilon}+\tau_{h}\right), \nabla\left(T^{\varepsilon}+\tau_{h}\right)\right)_{\varepsilon k}= \\
=c\left(a^{\varepsilon k} \nabla\left(T^{\varepsilon}+\tau_{h}\right), \nabla R_{t}^{\varepsilon}\right)_{\varepsilon k}, \\
\alpha_{\varepsilon}\left(u^{\varepsilon}, R_{t}^{\varepsilon} \nabla\left(T^{\varepsilon}+\tau_{h}\right)\right)_{\varepsilon f}=\alpha_{\varepsilon}\left(u^{\varepsilon}, R_{t}^{\varepsilon} \nabla R_{t}^{\varepsilon}\right)_{\varepsilon f}=0,
\end{gathered}
$$


and

it follows from (3.16) that

$$
\varepsilon \int_{\Gamma_{\varepsilon}} \eta^{\varepsilon}\left[T^{\varepsilon}\right]\left[R_{t}^{\varepsilon}\right] \geq 0
$$

$$
\left|R_{t}^{\varepsilon}\right|_{L^{4}\left(\Omega_{\varepsilon}^{t}\right)} \leq c\left|\left(Q, R_{t}^{\varepsilon}\right)\right| \leq|Q|_{L^{\infty}(\Omega)}\left|\Omega_{\varepsilon}^{t}\right|^{\frac{3}{4}}\left|R_{t}^{\varepsilon}\right|_{L^{4}\left(\Omega_{\varepsilon}^{t}\right)} .
$$

Moreover, considering $h>0$ we notice that

$$
\left|R_{t}^{\varepsilon}\right|^{4} \geq h^{4} \text { a.e. on } \Omega_{\varepsilon}^{t+h}
$$

and hence

$$
h\left|\Omega_{\varepsilon}^{t+h}\right|^{\frac{1}{4}} \leq\left|R_{t}^{\varepsilon}\right|_{L^{4}\left(\Omega_{\varepsilon}^{t+h}\right)} \leq\left|R_{t}^{\varepsilon}\right|_{L^{4}\left(\Omega_{\varepsilon}^{t}\right)} .
$$

Defining the nonincreasing function $\Phi_{\varepsilon}: \mathbb{R}_{+} \rightarrow \mathbb{R}_{+}$by $\Phi_{\varepsilon}(t)=\left|\Omega_{\varepsilon}^{t}\right|$, we see that

$$
\Phi_{\varepsilon}(t+h) \leq \frac{c}{h^{4}}|Q|_{L^{\infty}(\Omega)}^{4} \Phi_{\varepsilon}^{3}(t), \quad \forall t, h>0 .
$$

Applying Lemma B.1 (see [9], Chap. 2) it follows that there exists $c>0$ (independent of $\varepsilon$ ) such that $\Phi_{\varepsilon}\left(c|Q|_{L^{\infty}(\Omega)}\right)=0$, that is

$$
\operatorname{meas}\left\{x \in \Omega,\left|T^{\varepsilon}(x)+\tau_{h}(x)\right|>1 / 2+c|Q|_{L^{\infty}(\Omega)}\right\}=0,
$$

and the proof is completed.

Remark 3.5. We do not have a uniqueness result, except if we assume that the Rayleigh number $\alpha_{\varepsilon}>0$ is small enough.

\section{Compactness results for bounded Rayleigh numbers}

From now on we assume that there exists $\alpha>0$ such that

$$
\alpha_{\varepsilon} \rightarrow \alpha \text { when } \varepsilon \rightarrow 0 .
$$

First of all, let us notice that in the present case $h$ and $\tau_{h}$ are independent of $\varepsilon$.

We proceed with the a priori estimates of the solutions.

Setting $(v, S)=\left(u^{\varepsilon}, T^{\varepsilon}\right)$ in (3.15)-(3.16) and using (2.14)-(2.18) and (3.20), we get

$$
\left|u^{\varepsilon}\right|_{\varepsilon f} \leq c, \quad \varepsilon\left|\nabla u^{\varepsilon}\right|_{\varepsilon f} \leq c \text { and }\left|T^{\varepsilon}\right|_{H_{0}^{1}\left(\Omega \backslash \Gamma_{\varepsilon}\right)} \leq c,
$$

for some $c$ independent of $\varepsilon$.

For any $k \in\{s, f\}$, let us introduce the following notations

$$
\begin{gathered}
V_{0}(\operatorname{div}, \Omega)=\{v \in H(\operatorname{div}, \Omega), \operatorname{div} v=0 \text { in } \Omega, v \cdot \nu=0 \text { on } \partial \Omega\} \\
H_{\mathrm{per}}^{1}\left(Y_{k}\right)=\left\{\varphi \in H_{\mathrm{loc}}^{1}\left(\mathbb{R}_{k}^{N}\right), \varphi \text { is } Y \text {-periodic }\right\} \\
V_{0}\left(Y_{f}\right)=\left\{v \in H_{\mathrm{per}}^{1}\left(Y_{f}\right)^{N}, \operatorname{div} v=0 \text { in } Y_{f}, v=0 \text { on } \Gamma\right\} . \\
\tilde{H}_{\mathrm{per}}^{1}\left(Y_{k}\right)=\left\{\varphi \in H_{\mathrm{per}}^{1}\left(Y_{k}\right), \quad \tilde{\varphi}=0\right\},
\end{gathered}
$$

where for any $\varphi \in L^{1}(\Omega \times Y)$, we denote

$$
\tilde{\varphi}=\int_{Y} \varphi d y .
$$

The estimates (4.2) prove that $\chi_{f}^{\varepsilon}\left(u^{\varepsilon}\right), \varepsilon \chi_{f}^{\varepsilon}\left(\nabla u^{\varepsilon}\right)$ and $\chi_{k}^{\varepsilon}\left(T^{\varepsilon}\right), \varepsilon \chi_{k}^{\varepsilon}\left(\nabla T^{\varepsilon}\right)$ are respectively bounded in $L^{2}(\Omega)^{N}$ and $L^{2}(\Omega)$, for any $k \in\{s, f\}$. A first compactness result follows:

Theorem 4.1. There exist $u \in L^{2}\left(\Omega ; V_{0}\left(Y_{f}\right)\right), T_{k} \in H_{0}^{1}(\Omega)$ and $S_{k} \in L^{2}\left(\Omega ; \tilde{H}_{\mathrm{per}}^{1}\left(Y_{k}\right)\right), k \in\{s, f\}$, with the properties:

$$
\begin{gathered}
\chi_{f}^{\varepsilon}\left(u^{\varepsilon}\right) \stackrel{2 s}{\rightarrow} \chi_{f}(u), \\
\varepsilon \chi_{f}^{\varepsilon}\left(\frac{\partial u^{\varepsilon}}{\partial x_{i}}\right) \stackrel{2 s}{\rightarrow} \chi_{f}\left(\frac{\partial u}{\partial y_{i}}\right),
\end{gathered}
$$




$$
\begin{gathered}
\chi_{k}^{\varepsilon}\left(T^{\varepsilon}\right) \stackrel{2 s}{\rightarrow} \chi_{k}\left(T_{k}\right), \\
\chi_{k}^{\varepsilon}\left(\frac{\partial T^{\varepsilon}}{\partial x_{i}}\right) \stackrel{2 s}{\rightarrow} \chi_{k}\left(\frac{\partial T_{k}}{\partial x_{i}}+\frac{\partial S_{k}}{\partial y_{i}}\right), \quad i \in\{1,2, \ldots, N\} .
\end{gathered}
$$

where $\stackrel{2 s}{\rightarrow}$ represents the two-scale convergence in $L^{2}(\Omega)$. Moreover, we have:

$$
\tilde{u} \in V_{0}(\operatorname{div}, \Omega) .
$$

Proof. All the properties can be easily obtained by applying Proposition 1.14 of [1]. We only prove here that $T_{k}=0$ on $\partial \Omega$, for both $k \in\{s, f\}$ and $u=0$ on $\Omega \times \Gamma$.

Recalling the extension operators introduced by Lemma 2.1, we have

$$
\left|P_{\varepsilon k} T_{k}^{\varepsilon}\right|_{H_{0}^{1}(\Omega)} \leq c\left|\nabla P_{\varepsilon k} T_{k}^{\varepsilon}\right|_{L^{2}(\Omega)} \leq c\left|\nabla T_{k}^{\varepsilon}\right|_{L^{2}\left(\Omega_{\varepsilon k}\right)} \leq c
$$

which shows that $\left(P_{\varepsilon k} T_{k}^{\varepsilon}\right)_{\varepsilon}$ is bounded in $H_{0}^{1}(\Omega)$. Hence, there exists $T_{k}^{\prime} \in H_{0}^{1}(\Omega)$ such that

and consequently

$$
P_{\varepsilon k} T_{k}^{\varepsilon} \longrightarrow T_{k}^{\prime} \text { weakly in } H_{0}^{1}(\Omega),
$$

$$
\chi_{k}\left(\left\{\frac{x}{\varepsilon}\right\}\right) P_{\varepsilon k} T_{k}^{\varepsilon} \stackrel{2 s}{\rightarrow} \chi_{k}(y) T_{k}^{\prime}(x) .
$$

On the other hand,

$$
\chi_{k}\left(\left\{\frac{x}{\varepsilon}\right\}\right) P_{\varepsilon k} T_{k}^{\varepsilon}=\chi_{k}^{\varepsilon}\left(T_{k}^{\varepsilon}\right) \stackrel{2 s}{\rightarrow} \chi_{k}(y) T_{k}(x)
$$

which obviously implies $T_{k}=T_{k}^{\prime} \in H_{0}^{1}(\Omega)$.

Next, let $\varphi \in \mathcal{D}\left(\Omega, \mathcal{C}_{\text {per }}^{\infty}\left(\bar{Y}_{f}\right)\right)^{N}$. Denoting $\varphi^{\varepsilon}(x)=\varphi\left(x, \frac{x}{\varepsilon}\right)$, it follows

$$
\begin{gathered}
0=\varepsilon \int_{\Gamma_{\varepsilon}} u_{i}^{\varepsilon}\left(\varphi^{\varepsilon} \cdot \nu^{\varepsilon}\right)=\int_{\Omega_{\varepsilon f}}\left(\left(\varepsilon \nabla u_{i}^{\varepsilon}\right) \varphi^{\varepsilon}+u_{i}^{\varepsilon}\left(\varepsilon \operatorname{div} \varphi^{\varepsilon}\right)\right) \rightarrow \\
\rightarrow \int_{\Omega \times Y_{f}}\left(\varphi \nabla_{y} u_{i}+u_{i} \operatorname{div}_{y} \varphi\right)=\int_{\Omega \times Y_{f}} \operatorname{div}_{y}\left(u_{i} \varphi\right)=\int_{\Omega \times \Gamma} u_{i}(\varphi \cdot \nu)
\end{gathered}
$$

and the result follows.

Remark 4.2. There is in the previous proof a second interpretation of the two limit temperatures, $T_{s}$ and $T_{f}$; they are the weak limits of respectively $\left(P_{\varepsilon s} T_{s}^{\varepsilon}\right)_{\varepsilon}$ and $\left(P_{\varepsilon f} T_{f}^{\varepsilon}\right)_{\varepsilon}$ in $H_{0}^{1}(\Omega)$.

In order to study the asymptotic behaviour of $\left(u^{\varepsilon}, T^{\varepsilon}\right)$, we have to recover and estimate the pressure, which was hidden by the variational formulation. For this, let us define $F_{\varepsilon} \in H^{-1}(\Omega)$ by

$$
F_{\varepsilon}(v)=-\varepsilon^{2}\left(\nabla u^{\varepsilon}, \nabla v\right)_{\varepsilon f}+\left(\left(T^{\varepsilon}+\tau_{h}\right) g, v\right)_{\varepsilon f}, \quad v \in H_{0}^{1}\left(\Omega_{\varepsilon f}\right) .
$$

As $F_{\varepsilon}(v)=0$ if $\operatorname{div} v=0$ in $\Omega_{\varepsilon f}$, from the Tartar's variant of the De Rham Lemma (see Remark 1.9 [18]), we see that there exists $p^{\varepsilon} \in L_{0}^{2}\left(\Omega_{\varepsilon f}\right)$ such that

$$
F_{\varepsilon}(v)=\left\langle\nabla p^{\varepsilon}, v\right\rangle_{\left\langle H^{-1}, H_{0}^{1}\right\rangle\left(\Omega_{\varepsilon f}\right)}, \quad \forall v \in H_{0}^{1}\left(\Omega_{\varepsilon f}\right),
$$

where $L_{0}^{2}\left(\Omega_{\varepsilon f}\right)=\left\{p \in L^{2}\left(\Omega_{\varepsilon f}\right), \int_{\Omega_{\varepsilon f}} p=0\right\}$, that is

$$
\varepsilon^{2}\left(\nabla u^{\varepsilon}, \nabla v\right)_{\varepsilon f}-\left(p^{\varepsilon}, \operatorname{div} v\right)_{\varepsilon f}=\left(\left(T^{\varepsilon}+\tau_{h}\right) g, v\right)_{\varepsilon f}, \quad \forall v \in H_{0}^{1}\left(\Omega_{\varepsilon f}\right) .
$$

From (4.19) and Theorem 3.1 of [15] easily follows

$$
\left|\nabla p^{\varepsilon}\right|_{H^{-1}\left(\Omega_{\varepsilon f}\right)} \leq c \varepsilon \quad \text { and } \quad\left|p^{\varepsilon}\right|_{\varepsilon f} \leq c,
$$

for some $c>0$ independent of $\varepsilon$.

Thus, the hypotheses of Theorem 3.2 of [15] are fullfilled and the second final compactness result can be presented.

Theorem 4.3. There exists $p \in L_{0}^{2}(\Omega)=\left\{p \in L^{2}(\Omega), \quad \int_{\Omega} p=0\right\}$ such that on some subsequence

$$
\chi_{f}^{\varepsilon}\left(p^{\varepsilon}\right) \stackrel{2 s}{\rightarrow} \chi_{f}(y) p(x)
$$




\section{The homogenized system}

Now, we shall look for the two-scale system verified by the limits $u, p, T_{k}$ and $S_{k}, k \in\{s, f\}$, introduced by the theorems 4.1 and 4.3 .

Theorem 5.1. For any $\psi \in \mathcal{D}\left(\Omega ; V_{0}\left(Y_{f}\right)\right), \Phi_{k} \in \mathcal{D}(\Omega)$ and $\varphi_{k} \in \mathcal{D}\left(\Omega ; \tilde{H}_{\mathrm{per}}^{1}\left(Y_{k}\right)\right)$ we have

$$
\begin{gathered}
\int_{\Omega \times Y_{f}} \nabla_{y} u \nabla_{y} \psi-\int_{\Omega \times Y_{f}} p \operatorname{div}_{x} \psi=\int_{\Omega \times Y_{f}}\left(T_{f}+\tau_{h}\right) g \psi \\
\sum_{k \in\{s, f\}} \int_{\Omega \times Y_{k}} a^{k}(y)\left(\nabla T_{k}+\nabla_{y} S_{k}\right)\left(\nabla \Phi_{k}+\nabla_{y} \varphi_{k}\right)+\int_{\Omega \times \Gamma} \eta\left(T_{s}-T_{f}\right)\left(\Phi_{s}-\Phi_{f}\right)+ \\
+m \alpha \int_{\Omega} \tilde{u} \Phi_{f} \nabla\left(T_{f}+\tau_{h}\right)=\sum_{k \in\{s, f\}} \int_{\Omega \times Y_{k}} Q \Phi_{k},
\end{gathered}
$$

where $\left.m:=\left|Y_{f}\right| \in\right] 0,1[$.

Proof. We pass (4.20) and (3.16) to the limit $(\varepsilon \rightarrow 0)$ with specific test functions. In (4.20) we set:

$$
v(x)=\psi^{\varepsilon}(x)=\psi(x, x / \varepsilon) \text { for } x \in \Omega_{\varepsilon f}, \quad \psi \in \mathcal{D}\left(\Omega ; V_{0}\left(Y_{f}\right)\right)
$$

and in (3.16) we set

$$
S(x)=\Phi_{k}(x)+\varepsilon \varphi_{k}^{\varepsilon}(x) \text { for } x \in \Omega_{\varepsilon k}, k \in\{s, f\},
$$

where $\Phi_{k} \in \mathcal{D}(\Omega)$ and $\varphi_{k}^{\varepsilon}(x)=\varphi(x, x / \varepsilon)$ for $x \in \Omega_{\varepsilon k}, \varphi_{k} \in \mathcal{D}\left(\Omega ; \mathcal{C}_{\text {per }}^{\infty}\left(Y_{k}\right)\right)$. Letting $\varepsilon \rightarrow 0$ on the subsequence ensured by the theorems $4.1-4.3$ we find without difficulty (5.1); with the techniques of [6] and [8] we prove the convergences to the all the terms of (5.2), except the nonlinear one, which we treat here. Recalling Remark 4.2, as $P_{\varepsilon f} T^{\varepsilon}$ is strongly converging to $T_{f}$ in $L^{2}(\Omega)$, we have

$$
\begin{gathered}
\alpha_{\varepsilon} \int_{\Omega_{\varepsilon f}} u^{\varepsilon} \Phi_{f} \nabla\left(T^{\varepsilon}+\tau_{h}\right)=-\alpha_{\varepsilon} \int_{\Omega} \chi_{f}^{\varepsilon}\left(u^{\varepsilon}\right)\left(P_{\varepsilon f} T^{\varepsilon}+\tau_{h}\right) \nabla \Phi_{f} \rightarrow \\
\rightarrow-\alpha \int_{\Omega \times Y_{f}} u\left(T_{f}+\tau_{h}\right) \nabla \Phi_{f}=m \alpha \int_{\Omega} \tilde{u} \Phi_{f} \nabla\left(T_{f}+\tau_{h}\right)
\end{gathered}
$$

Using the estimations (4.2), the other nonlinear term is converging to zero, as follows:

$$
\left|\varepsilon \alpha_{\varepsilon} \int_{\Omega_{\varepsilon f}} u^{\varepsilon} \varphi_{f}^{\varepsilon} \nabla\left(T^{\varepsilon}+\tau_{h}\right)\right| \leq C \varepsilon\left|u^{\varepsilon}\right|_{L^{2}\left(\Omega_{\varepsilon f}\right)}\left|\nabla\left(T^{\varepsilon}+\tau_{h}\right)\right|_{L^{2}\left(\Omega_{\varepsilon f}\right)} \sup _{\Omega \times Y}\left|\varphi_{f}\right| .
$$

Moreover, (5.2) holds true for any $\varphi_{k} \in \mathcal{D}\left(\Omega ; \tilde{H}_{\text {per }}^{1}\left(Y_{k}\right)\right)$ by density and continuity arguments.

The next result is straightforward.

Theorem 5.2. The limits $u$ and $S_{k}$ of the theorems 4.1-4.3 are uniquely determined with respect to $p$ and $T_{k}$ by the following relations:

$$
\begin{gathered}
u_{j}(x, y)=w_{j}^{(i)}(y)\left(\left(T_{f}+\tau_{h}\right) g_{i}-\frac{\partial p}{\partial x_{i}}\right)(x) \text { in } \Omega \times Y_{f}, \\
S_{k}(x, y)=S_{k}^{(i)}(y) \frac{\partial T_{k}}{\partial x_{i}}(x) \text { in } \Omega \times Y_{k}, \quad k \in\{s, f\},
\end{gathered}
$$

where $w^{(i)} \in V_{0}\left(Y_{f}\right)$ and $S_{k}^{(i)} \in \tilde{H}_{\mathrm{per}}^{1}\left(Y_{f}\right)$ are the solutions of the well-defined (local) problems:

$$
\begin{aligned}
\int_{Y_{f}} \nabla w^{(i)} \nabla \psi & =\int_{Y_{f}} \psi_{i}, \quad \forall \psi \in V_{0}\left(Y_{f}\right), \\
\int_{Y_{k}} a^{k} \nabla\left(S_{k}^{(i)}+y_{i}\right) \nabla \psi_{k} & =0, \quad \forall \psi_{k} \in \tilde{H}_{\mathrm{per}}^{1}\left(Y_{k}\right), \quad k \in\{s, f\} .
\end{aligned}
$$

Remark 5.3. Denoting with $B$ the inverse of the positive-definite matrix $\left(\int_{Y_{f}} \nabla w^{(i)} \nabla w^{(j)}\right)_{i, j} \in \mathbb{R}^{N \times N}$, we get from (5.4)

$$
B \tilde{u}+\nabla p=\left(T_{f}+\tau_{h}\right) g \text { in } \Omega .
$$


Remark 5.4. Introducing for $k \in\{s, f\}$ the positive-definite matrices

$$
A_{i j}=\int_{Y_{k}} a^{k} \nabla\left(S_{k}^{(i)}+y_{i}\right) \nabla\left(S_{k}^{(j)}+y_{j}\right)
$$

by taking into account (5.5) in (5.2), we obtain:

where $\hat{\eta}=\int_{\Gamma} \eta d \sigma$.

$$
\begin{gathered}
\sum_{k \in\{s, f\}}\left(A^{k} \int_{\Omega} \nabla T_{k} \nabla \Phi_{k}\right)+\hat{\eta} \int_{\Omega}\left(T_{s}-T_{f}\right)\left(\Phi_{s}-\Phi_{f}\right)+ \\
+m \alpha \int_{\Omega} \tilde{u} \Phi_{f} \nabla\left(T_{f}+\tau_{h}\right)=\sum_{k \in\{s, f\}} \int_{\Omega \times Y_{k}} Q \Phi_{k},
\end{gathered}
$$

Returning to the initial notations, that is, $\theta_{k}=T_{k}+\tau_{h}, k \in\{s, f\}$, and summarizing the results of the theorems 4.1-4.3 and of the remarks 5.3-5.4, we see that the homogenized system corresponding to $(3.5)-(3.11)$ is the following:

$$
\begin{gathered}
\operatorname{div} \tilde{u}=0 \quad \text { in } \Omega, \\
B \tilde{u}+\nabla p=\theta_{f} g \quad \text { in } \Omega, \\
m \alpha \tilde{u} \nabla \theta_{f}-\operatorname{div}\left(A^{f} \nabla \theta_{f}\right)+\hat{\eta}\left(\theta_{f}-\theta_{s}\right)=m Q \quad \text { in } \Omega, \\
-\operatorname{div}\left(A^{s} \nabla \theta_{s}\right)+\hat{\eta}\left(\theta_{s}-\theta_{f}\right)=(1-m) Q \quad \text { in } \Omega, \\
\tilde{u} \cdot n=0 \quad \text { on } \partial \Omega, \\
\theta_{f}=\theta_{s}=\tau \quad \text { on } \partial \Omega .
\end{gathered}
$$

Like the initial system, it has at least one weak solution, which is unique only when $\alpha>0$ is sufficiently small.

\section{Acknowledgment}

This work has been accomplished during the visit of D. Poliševski at the I.R.M.A.R.'s Department of Mechanics (University of Rennes 1), whose support is gratefully acknowledged.

\section{References}

[1] Allaire, G., Homogenization and two-scale convergence, S.I.A.M. J. Math. Anal., 23 (1992), 1482-151.

[2] Barenblatt, G.I., Zheltov, Y.P., Kochina, I.N., On basic conceptions of the theory of homogeneous fluids seepage in fractured rocks (in Russian), Prikl.Mat. i Mekh., 24 (1960), 852-864.

[3] Cioranescu, D., Donato, P., An Introduction to Homogenization, Oxford Lecture Series in Mathematics and Its Applications, vol. 17, Oxford Univ. Press, New York, 1999.

[4] Cioranescu, D., Saint Jean-Paulin, J., Homogenization in open sets with holes, J. Math. Anal. Appl. 71(2) (1979), 590-607.

[5] Duval, F., Fichot, F., Quintard, M., A local thermal non-equilibrium model for two-phase flows with phase-change in porous media, Int. J. Heat Mass Transfer, 47 (2004), 613-639.

[6] Ene, H.I., Poliševski, D., Model of diffusion in partially fissured media, Z.A.M.P. 53 (2002), 1052-1059.

[7] Gossez, J.P., Remarques sur les Opérateurs Monotones, Bull. Cl. Sc. Acad. Roy. Belg. 9 (1966), 1073-1077.

[8] Gruais I., Poliševski, D., Heat transfer models for two-component media with interfacial jump, Appl. Anal., 96(2) (2017), 247-260.

[9] Kinderlehrer, D., Stampacchia, G., An introduction to Variational Inequalities and Their Applications, Academic Press, New-York, 1980.

[10] Nguetseng, G., A general convergence result for a functional related to the theory of homogenization, S.I.A.M. J.Math.Anal. 20 (1989), 608-623.

[11] Nield, D.A., Bejan, A., Convection in porous media. 2d Edition, ( Chap. 2 and Chap. 6), Springer-Verlag, New-York, 1999.

[12] Poliševski, D., Weak continuity in convection problems, J. Math. Anal. Appl. 110(1) (1985), 51-58. 
[13] Ene, H.I., Poliševski, D., Thermal Flow in Porous Media, D. Reidel Pub. Co., Dordrecht, 1987.

[14] Poliševski, D., Homogenization of thermal flows: the influence of Grashof and Prandtl numbers, Int. J. Engng. Sci. 28(4) (1990), 285-291.

[15] Poliševski, D., Basic homogenization results for a biconnected $\varepsilon$-periodic structure, Appl. Anal. 82(4) (2003), 301-309.

[16] Quintard, M., Modelling local non-equilibrium heat transfer in porous media, Proc. 11th Int. Heat Transfer Conf., 1 (1998), 279-285.

[17] Rees, A.S., Pop I., Local thermal non-equilibrium in porous medium convection, in Transport phenomena in porous media III, D.B. Ingham and I. Pop (eds.), Elsevier, Oxford, 2005, 147-173.

[18] Temam, R., Navier-Stokes Equations. Theory and Numerical Analysis, AMS Chelsea Publishing, Providence, Rhode Island, 2001.

[19] Vortmeyer, D., Schaefer, R.J., Equivalence of one- and two-phase models for heat transfer processes in packed beds, one dimensional theory, Chem. Eng. Sci., 29 (1974), 485-491.

[20] Whitaker, S., The method of volume averaging., Kluwer, Dordrecht, 1999.

\section{Isabelle Gruais}

Université de Rennes 1, I.R.M.A.R

Campus de Beaulieu

35042 Rennes Cedex

France

e-mail: isabelle.gruais@univ-rennes1.fr

Dan Poliševski

Inst. Math. "S.Stoilow"

Romanian Academy

P.O. Box 1-764

Bucharest

Romania 